\title{
Impact of educational intervention on medication adherence, quality of life, knowledge attitude and practice among benign prostate hyperplasia patients: a prospective study
}

\author{
Ashlin Treesa Johnson, Vineeth Jayakumar*, Pritty Anna Varghese, \\ Karthik Vijayan, Babitha Martin
}

Sree Krishna College of Pharmacy and Research Center, Parassala, Thiruvananthapuram, Kerala, India

Received: 01 October 2020

Revised: 01 November 2020

Accepted: 02 November 2020

\section{*Correspondence:}

Vineeth Jayakumar,

Email: vineethjayakumar@gmail.com

Copyright: (c) the author(s), publisher and licensee Medip Academy. This is an open-access article distributed under the terms of the Creative Commons Attribution Non-Commercial License, which permits unrestricted non-commercial use, distribution, and reproduction in any medium, provided the original work is properly cited.

\begin{abstract}
Background: Benign prostate hyperplasia (BPH) is one of the common bothersome diseases among elderly men of age greater than 50 years. The aim of the current study was to evaluate the impact of patient counselling on quality of life (QoL), knowledge, attitude and practice (KAP) and medication adherence among patients.

Methods: A Prospective study was conducted in a total of $110 \mathrm{BPH}$ patients from the department of urology for a period of 6 months. 55 patients in each group received silodosin $8 \mathrm{mg}$ or tamsulosin $0.4 \mathrm{mg}$ once daily. Data was collected by using a suitably designed proforma, international prostate symptom score (IPSS) and BPH impact index were used for assessing QoL, morisky green levin scale for medication adherence and a suitably designed KAP questionnaire for assessing KAP. Patients were counselled regarding the disease, drugs and lifestyle modifications using patient information leaflet.

Results: The current study found that both the Tamsulosin and Silodosin group had an improvement on QoL, IPSS Q 8 by $36 \%$ and $41.5 \%$ respectively and improvement on BII by $35.5 \%$ and $47.3 \%$ respectively. Medication adherence had significant improvement from first to second review by $12 \%$. Significant improvement in knowledge by $80.1 \%$, attitude by $72.7 \%$ and practice by $79.2 \%$ were observed.

Conclusions: The provision of effective counselling was found to have a profound impact on improving patient's quality of life, medication adherence, knowledge, attitude and practice.
\end{abstract}

Keywords: Benign prostate hyperplasia, International prostate symptom score, Morisky green levin, Adherence, Knowledge, Attitude and practice

\section{INTRODUCTION}

Benign prostatic hyperplasia (BPH) is the most common benign tumor in men and is responsible for urinary symptoms in majority of males over the age of 50 years. ${ }^{1}$ This refers to the proliferation of smooth muscles and epithelial cells within the prostatic transition zone. ${ }^{2}$ It is a complex disease and is often associated with lower urinary tract symptoms (LUTS) which includes nocturia, urgency, urinary frequency, urinary tract infections, benign prostatic obstruction. Prevalence and severity of LUTS in the aging male can be progressive and is an important diagnosis in the health care of patients and welfare of society. ${ }^{1}$

Autopsy studies have revealed the histological presence of $\mathrm{BPH}$ in $50 \%$ of males aged 51-60 years, increasing to $90 \%$ in those over 85 yrs. By the age of 80 years, 
virtually all men exhibit one or more of the symptoms associated with BPH. BPH is seen in all races although the overall size of the prostate varies from race to race. ${ }^{3}$

Prostatic hypertrophy is directly related to the aging process and to the hormonal activity. Within the prostate, testosterone is converted by 5 alpha -reductase to dihydrotestosterone (DHT). DHT is five times more potent than testosterone and is responsible for stimulating growth factor that influence cell division leading to prostatic hyperplasia and enlargement. ${ }^{4}$

LUTSs can be divided into symptoms caused by failure of urine storage (irritative) and those caused by failure to empty the bladder (obstructive or voiding). Irritative symptoms include frequency, urgency, nocturia and obstructive symptoms include straining, intermittency, weak stream and incomplete emptying. ${ }^{5}$

The range of treatment options for the management of $\mathrm{BPH}$ includes watchful waiting, medical therapies and surgical interventions. ${ }^{6}$ Drug treatment goals for benign prostatic hyperplasia includes relieving obstructive and irritative voiding symptoms, preventing complications of disease and reducing the need for surgical intervention. ${ }^{7}$ Watchful therapy is recommended for patients with mild symptoms, medical treatment for patients with mild to moderate symptoms, and surgery for patients who failed medication/ conservative management and who have moderate to severe symptoms or who have complications of $\mathrm{BPH}^{8}$ Therapeutic management includes alpha blockers, $5 \alpha$-reductase inhibitors or combination of both and phytotherapy. The widely used drugs for BPH are tamsulosin, silodosin, finasteride and dutasteride. ${ }^{9}$

Patient counselling refers to the process of providing information, advice and assistance to help patients to use their medications appropriately. The information and advice are given by the pharmacist directly to the patient or to the patient's representatives, and may also include information about the patient's illness or recommended lifestyle changes.

WHO defines quality of life (QoL) as an individual's perception of their position in life in the context of the culture and value systems in which they live and in relation to their goals, expectations, standards and concerns. Medication adherence is defined as the extent to which patient's medication taking behavior coincides with the intention of health advice he/she has been given. ${ }^{14}$

Patient counselling sessions provided during the study could help patients to have necessary knowledge, attitude and practice about the disease and could help to improve QoL and medication adherence. This study focuses on the impact of patient counselling on QoL, knowledge, attitude and practice (KAP) and medication adherence among BPH patients.

\section{METHODS}

A prospective study was conducted in patients from the department of Urology in Cosmopolitan Hospital, Trivandrum who were diagnosed with BPH during the study period after obtaining permission for collection of data from the institutional human ethical committee. The study was for a period of 6 months from December 2018 to May 2019. A written informed consent was taken from the patients diagnosed with BPH satisfying the inclusion and exclusion criteria.

\section{Inclusion criteria}

Inclusion criteria were 1) BPH patients who are willing to participate in the study from OP setting 2) patients of age greater than 50 years 3) international prostate symptom score (IPSS) $\leq 23$ who lacks absolute indication of surgical intervention

\section{Exclusion criteria}

Exclusion criteria were 1) patients with raised serum prostate specific antigen level (>20ngml)/suspected prostatic malignancy 2) post void residual urine of $>200 \mathrm{ml} \mathrm{3)}$ history of lower urinary tract malignancy/pelvic surgery 4) neurological conditions causing bladder dysfunction, hepato-renal insufficiency.

All information relevant to the study was collected from case records by using a suitably designed proforma and direct interview with patients using questionnaires- IPSS, BII, KAP, MGL. All the scales were translated into local language (Malayalam) and the patients were requested to fill them.

IPSS is a symptom severity assessing tool which comprise of eight questions, in which seven questions address the symptom severity and one question addresses the QoL. ${ }^{10} \mathrm{BPH}$ impact index is a tool used to assess the impact of lower urinary tract symptoms on patient with BPH. It includes a total of 4 questions. ${ }^{12}$ KAP questionnaire consists of 15 questions- out of which five are knowledge-based questions, five are attitude-based questions and five are practice based questions (annexure-I). Morisky green levine (MGL) questionnaire consists of four questions for assessing medication adherence. $^{11}$

Patients were requested to answer the questionnaire and were counselled regarding the disease, drugs and lifestyle modifications using patient information leaflet (PIL), which was provided in english and local language (Malayalam). The reliability of the PIL was calculated and verified using the flesch reading ease (FRE) formula.

The impact of patient counselling on QoL was assessed using I-PSS Q8 and BPH impact index at the first and second visits, medication adherence was assessed using MGL at the first and second review, knowledge, attitude 
\& practice of BPH patients was assessed using a suitably designed KAP questionnaire for $\mathrm{BPH}$ at first and second visits. Patients were asked for review after first and second month of taking the medication. At the end of the study, all the parameters and scores were compared from baseline to end of study.

For data entry we had used the software microsoft excel and all the analysis were carried out with the help of statistical software SPSS V.22 for WINDOWS. For the improvement of scores within the group, paired ' $t$ ' test have been used.

\section{RESULTS}

In our study, we analyzed the data collected from 110 patients with BPH at Urology department. 55 patients in each group, they received silodosin $8 \mathrm{mg}$ or tamsulosin 0.4 $\mathrm{mg}$ once daily. Out of the total sample size of 120 , there was 10 drop out as they were unable to come for followup or opted for surgical intervention. This study aimed to evaluate the impact of counseling on QoL, KAP and medication adherence among patients with BPH. The comparison of improvement before and after counselling was assessed statistically with paired ' $t$ '-test. A calculated p-value less than 0.05 was considered to be statistically significant.

\section{Demographic details of patients}

In this section, the data related to demographic details of the patients were collected and the calculated frequencies and percentages were reported. The distribution of total patients based on age from both groups have been shown in the (Table 1).

From table 1, it was observed that out of 110 patients 22 (20\%) were below 60 years of age, $58(52.7 \%)$ patients were in between 61-70 years, $23(20.9 \%)$ were in between $71-80$ years and $7(6.4 \%)$ were above $80 y$ rs of age. We found that more than $50 \%$ of patients attended the urology OPD was of age group 61-70 years.

The distribution of total patients based on literacy was classified into illiterate, primary/secondary education and degree is shown in Figure 1. From Figure 2, it was observed that out of 110 patients, $6(1.8 \%)$ patients were illiterate, $41(37.3 \%)$ patients were of primary or secondary level education and $63(57.2 \%)$ patients were degree holders. We inferred that about three fourth of patients were literate.

\section{Symptomatic distribution of $\mathrm{BPH}$ patients}

The symptomatic distribution of BPH patients was assessed using I-PSS and is shown in (Table 2). We observed that about $12.7 \%$ of patients were mildly symptomatic, $68.2 \%$ of patients were moderately symptomatic and $19.1 \%$ of patients were severely symptomatic. Thus, we inferred that more than half of the patients attending urology OP were moderately symptomatic.

Table 1: Frequency and percentage distribution of total no. of patients based on age.

\begin{tabular}{|l|ll|}
\hline Age (in years) & Frequency & Percentage \\
\hline$\leq \mathbf{6 0}$ & 22 & 20 \\
\hline $\mathbf{6 1 - 7 0}$ & 58 & 52.7 \\
\hline $\mathbf{7 1 - 8 0}$ & 23 & 20.9 \\
\hline $\mathbf{> 8 0}$ & 7 & 6.4 \\
\hline Total & 110 & 100 \\
\hline
\end{tabular}

Table 2: Symptomatic distribution of BPH patients.

\begin{tabular}{|lll|}
\hline Symptom severity & Frequency & Percentage \\
\hline Mildly symptomatic & 14 & 12.7 \\
\hline $\begin{array}{l}\text { Moderately } \\
\text { symptomatic }\end{array}$ & 75 & 68.2 \\
\hline Severely symptomatic & 21 & 19.1 \\
\hline
\end{tabular}

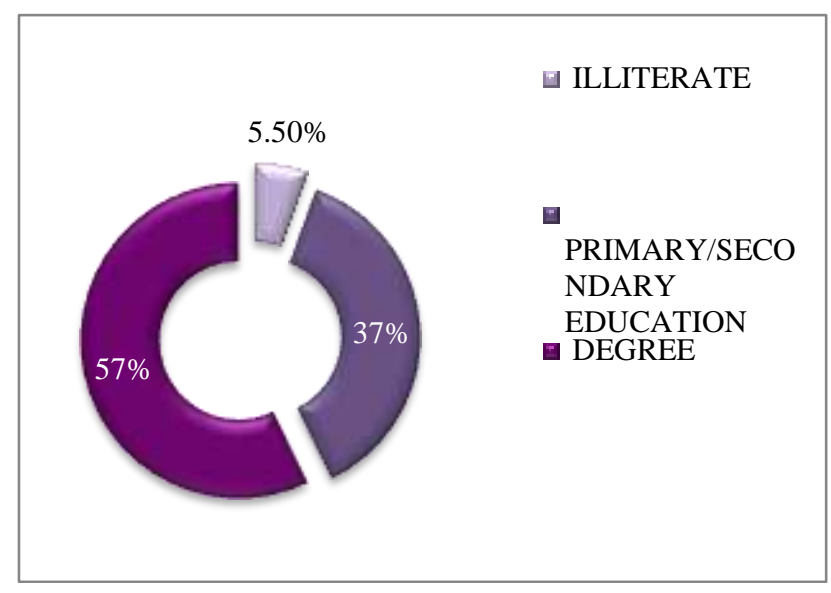

Figure 1: Percentage distribution of patients based on literacy.

\section{Impact of patient counselling on QoL.}

The impact of patient counselling on QoL was assessed using BPH impact index (BII) and I-PSS Q8 and was analyzed by paired ' $\mathrm{t}$ ' test.

From Figure 2, paired t test shows significant effect on improving patient's QoL in silodosin $8 \mathrm{mg}$ group by IPSS Q8 (' $\mathrm{t}$ '=16.15, $\mathrm{P}<0.01$ ) and BII (' $\mathrm{t}$ '=21.23, $\mathrm{p}<0.01$ ) as well as in tamsulosin $0.4 \mathrm{mg}$ group by IPSS Q8 (' $\mathrm{t}$ '=13.86, $\mathrm{p}<0.01$ ) and BII (' $\mathrm{t}$ '=27.48, $\mathrm{p}<0.01)$. Before the treatment, the IPSS Q8 score was $3.35 \pm 0.76$ (mostly dissatisfied) and BPH impact index was $6.85 \pm 2.18$ (moderate) in silodosin $8 \mathrm{mg}$ group and IPSS Q8 score was $3.36 \pm 1.02$ (mostly dissatisfied) and BPH Impact Index was $9.38 \pm 2.40$ (severe) in tamsulosin $0.4 \mathrm{mg}$ group. But after the treatment, it significantly improved to the values $1.96 \pm 0.90$ of IPSS Q8 score (mostly satisfied) and BII of $3.61 \pm 1.96$ (mild) in silodosin $8 \mathrm{mg}$ group and IPSS Q8 score of $2.27 \pm 0.97$ (mostly satisfied) and BII of 
$6.00 \pm 2.30$ (moderate) in tamsulosin $0.4 \mathrm{mg}$ group. Thus, patient counselling had a significant positive impact on patient's quality of life by $47.3 \%$ and $37.5 \%$ of reduction of BII in Silodosin and Tamsulosin groups and $41.5 \%$ and $36 \%$ reduction of IPSS Q8 in Silodosin and Tamsulosin group respectively.

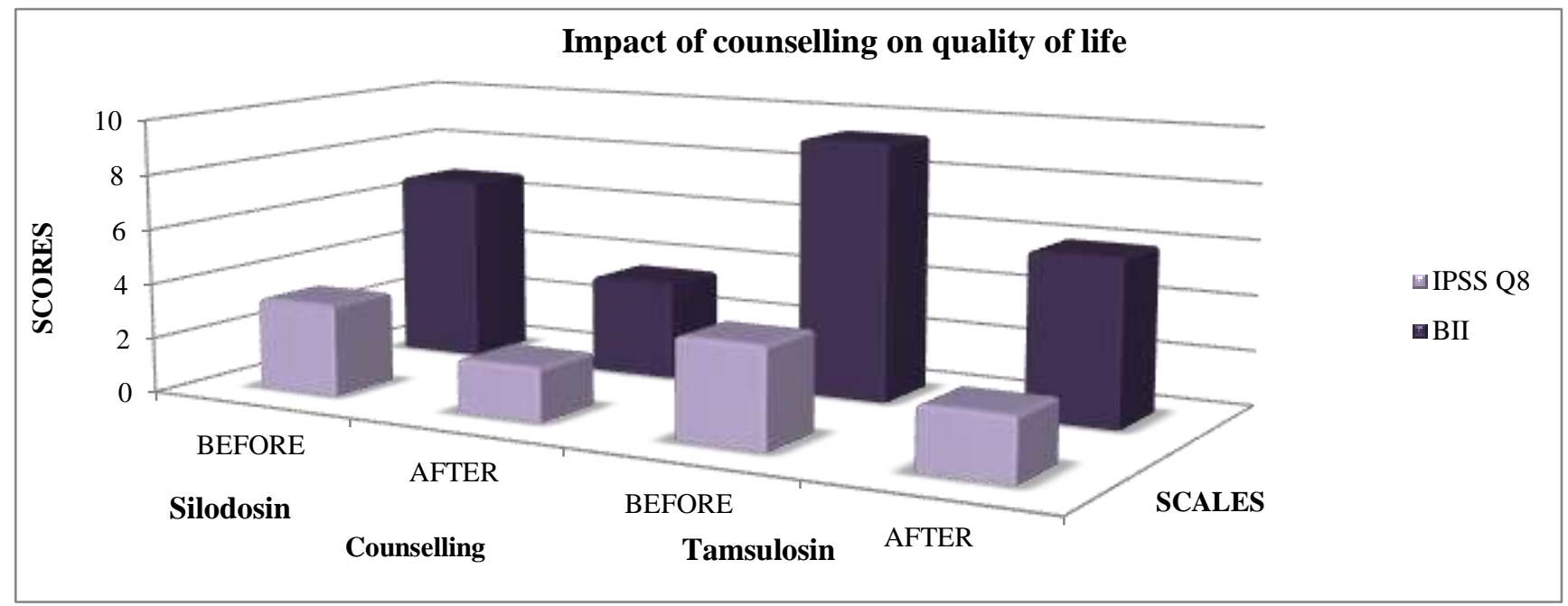

Figure 2: Effect of patient counselling on QoL in silodosin and tamsulosin groups.

Table 3: Effect of patient counselling on medication adherence in silodosin and tamsulosin groups.

\begin{tabular}{|c|c|c|c|c|c|c|}
\hline Drug & Review & Mean & SD & Percentage improvement & 't' & P value \\
\hline \multirow{2}{*}{ Silodosin } & $1^{\text {st }}$ & 2.70 & 0.98 & \multirow{2}{*}{88.5} & \multirow{2}{*}{20.43} & \multirow{2}{*}{0.000} \\
\hline & $2^{\text {nd }}$ & 0.31 & 0.60 & & & \\
\hline \multirow{2}{*}{ Tamsulosin } & $1^{\text {st }}$ & 2.30 & 0.87 & \multirow{2}{*}{93.9} & \multirow{2}{*}{21.00} & \multirow{2}{*}{0.000} \\
\hline & $2^{\text {nd }}$ & 0.14 & 0.35 & & & \\
\hline
\end{tabular}

Table 4: Effect of patient counselling on KAP in BPH patients (paired ' $t$ ' test).

\begin{tabular}{|c|c|c|c|c|c|c|}
\hline Parameter & Review & Mean & SD & Percentage improvement & ' $t$ ' & P value \\
\hline \multirow{2}{*}{ Knowledge } & $1^{\text {st }}$ & 2.64 & 2.79 & \multirow{2}{*}{80.1} & \multirow{2}{*}{27.52} & \multirow{2}{*}{0.000} \\
\hline & $2^{\text {nd }}$ & 13.28 & 1.92 & & & \\
\hline \multirow{2}{*}{ Attitude } & $1^{\text {st }}$ & 3.8 & 3.68 & \multirow{2}{*}{72.7} & \multirow{2}{*}{19.42} & \multirow{2}{*}{0.000} \\
\hline & $2^{\text {nd }}$ & 13.93 & 1.75 & & & \\
\hline \multirow{2}{*}{ Practice } & $1^{\text {st }}$ & 2.87 & 2.84 & \multirow{2}{*}{79.2} & \multirow{2}{*}{26.26} & \multirow{2}{*}{0.000} \\
\hline & $2^{\text {nd }}$ & 13.77 & 1.68 & & & \\
\hline
\end{tabular}

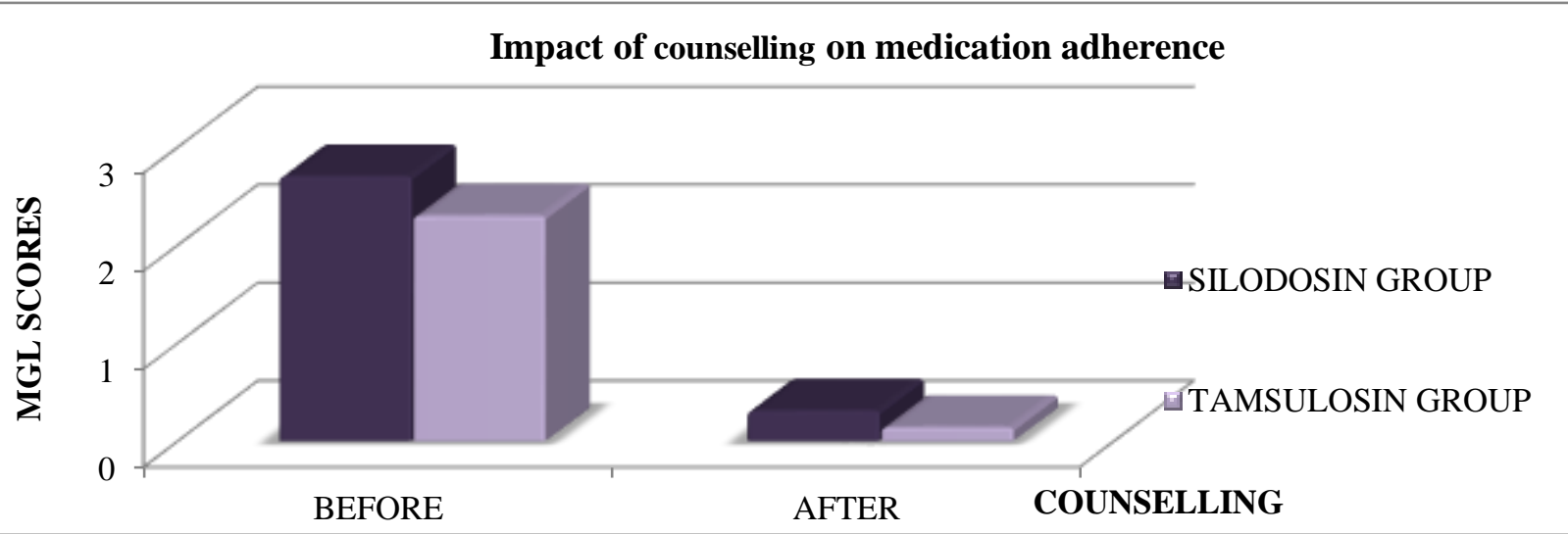

Figure 3: Diagrammatic representation of effect of patient counselling on medication adherence in silodosin and tamsulosin groups. 


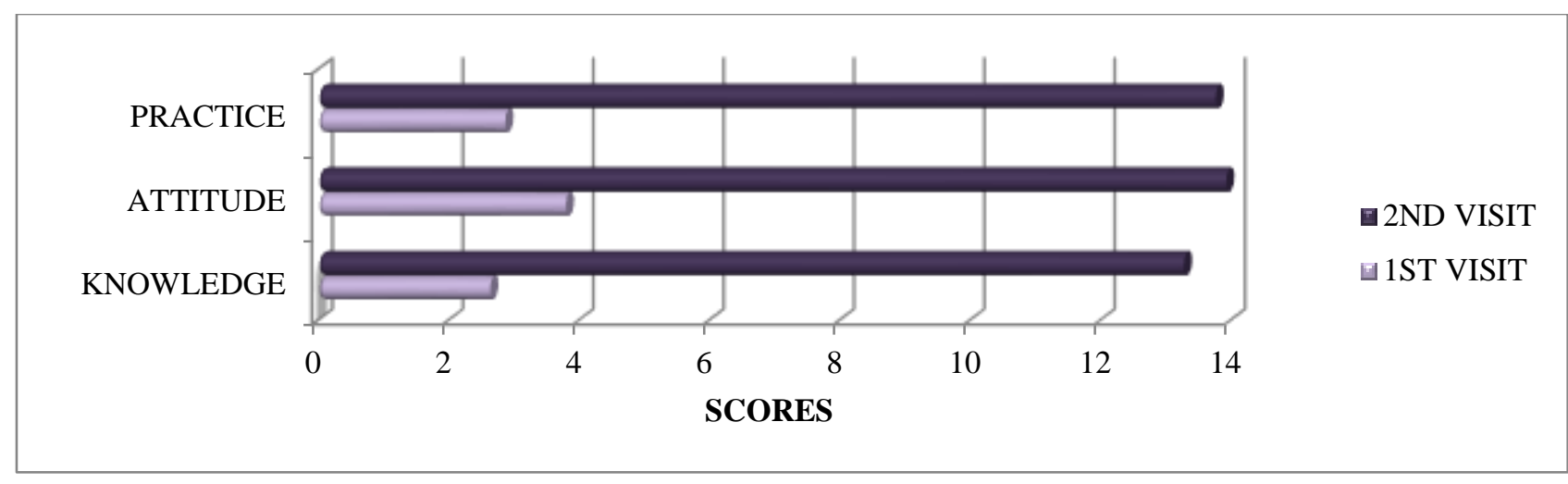

Figure 4: Diagrammatic representation of effect of patient counselling on KAP in BPH patients.

\section{Impact of patient counselling on medication adherence}

The impact of patient counselling on medication adherence was assessed using MGL questionnaire and was analyzed by paired $t$ test.

From (Table 3), paired ' $t$ ' test shows significant effect on improving medication adherence (measured using MGL) in Silodosin $8 \mathrm{mg}$ group (' $\mathrm{t}$ ' $=20.43, \mathrm{p}<0.01)$ as well as in tamsulosin $0.4 \mathrm{mg}$ group (' $\mathrm{t}$ ' $=21.00, \mathrm{p}<0.01)$. Before the treatment, the MGL score was $2.70 \pm 0.98$ (moderate) in silodosin $8 \mathrm{mg}$ group and 2.30 \pm 0.87 (severe) in tamsulosin $0.4 \mathrm{mg}$ group. But after the treatment, MGL significantly improved to the values $0.31 \pm 0.60$ (mild) in silodosin $8 \mathrm{mg}$ group by $88.5 \%$ and $0.14 \pm 0.35$ (moderate) in tamsulosin $0.4 \mathrm{mg}$ group by $93.9 \%$.

Thus, effective counselling had a positive impact on medication adherence by $88.5 \%$ in Silodosin group and 93.9\% in tamsulosin group (Figure 3).

\section{Impact of patient counselling on KAP}

The impact of patient counselling on KAP was assessed using validated KAP questionnaire and analyzed by paired ' $t$ ' test.

From (Table 4), we observed that the counselling made significant effect on improving patient's KAP (measured using KAP questionnaire) in BPH patients- [knowledge $(\mathrm{t}=27.5, \mathrm{p}<0.01)$, attitude $(\mathrm{t}=19.42, \mathrm{p}<0.01)$ and practice $(\mathrm{t}=26.26, \mathrm{p}<0.01)]$. Before counselling, the KAP levels were, $2.64 \pm 2.79$ (poor) for knowledge, $3.8 \pm 3.68$ (poor) for attitude and $2.87 \pm 2.84$ (poor) for practice. After counselling, KAP significantly improved to the values $13.28 \pm 1.92$ (good) for knowledge, 13.93 \pm 1.75 (good) for attitude, and $13.77 \pm 1.68$ (good) for practice.

It was proved that an effective counselling had a greater impact on knowledge by $80.1 \%$, attitude by $72.7 \%$ and practice by $79.2 \%$ (Figure 4 ). Thus, we inferred that structured and effective counselling can improve the patient's KAP.

\section{DISCUSSION}

$\mathrm{BPH}$ is the most common benign tumor in men and is responsible for LUTS and it is seen in majority of males over 50 years. LUTS increases with age in an overall prevalence greater than $50 \%$ in men of 50 years or older and are associated with a significant negative impact on patient's QOL as postulated by Mahajan et al. ${ }^{15}$ From our study, we found that about $52.7 \%$ of BPH patients attending the urology OP belonged to an age group of 6170 yrs. It is evident that as age increases the level of dihydrotestosterone and estrogen increases which could lead to stimulatory growth of prostate.

In the current study, we observed that more than half, that is $68.2 \%$ of the patients attending urology OP were moderately symptomatic. A study by Nagarthanm et al in a hospital at Andhra Pradesh also showed most of the BPH patients were moderately symptomatic. ${ }^{16}$

In our study we distributed total patients based on literacy as illiterate, primary/secondary education and degree levels. Out of total patients visited in urology OP majority were literate and among them, $57.2 \%$ were degree holders. A study conducted by Adepu et al in a tertiary care hospital at Karnataka states that $41.3 \%$ BPH patients were illiterate and $58.7 \%$ patients were literate. ${ }^{17}$

On the evaluation of impact of patient counselling on quality of life from our study, it was found that both the Tamsulosin and Silodosin group have improvement on IPSS Q8 by $36 \%$ and $41.5 \%$ respectively and improvement on BII by $35.5 \%$ and $47.3 \%$ respectively. This showed there was a positive impact on quality of life with counselling.

A study conducted by Adepu et al showed the influence of patient education on LUTS improvement and health related quality of life in patients with BPH. They concluded that patient counselling had a significant improvement in knowledge about the disease, symptoms, management of the disease, treatment outcomes and QoL of BPH patients. ${ }^{13,17}$ 
Our study showed a significant improvement in knowledge by $80.1 \%$, attitude by $72.7 \%$ and practice by $79.2 \%$. Even though they were literate, they had poor knowledge about the disease. So, there is a need of patient counselling to improve the health literacy among BPH patients. This study also showed significant improvement on KAP with effective counselling.

On the comparison of medication adherence within the group using MGL scale, we found a significant improvement from first to second review by $12 \%$. This showed significant improvement on medication adherence with the impact of effective counselling.

From the study, we proved that effective patient counselling could improve the health-related quality of life, KAP and medication adherence

One limitation of the study was, about a quarter of patients had received prior $\alpha$-blocker therapy, which may have impacted treatment differences, however, the reasons for patient's discontinuation of prior therapy were unknown.

\section{CONCLUSION}

$\mathrm{BPH}$ is known to be a bothersome disease in elderly men, mostly between 61 and 70 years of age. On assessing symptomatic distribution of patients, it was inferred that most of the outpatients who consulted the urology OP were moderately symptomatic. Patient counselling could significantly increase one's medication adherence, knowledge attitude and practice and hence QoL. Though adequate quality counselling practices by clinical pharmacists are available for variety of common diseases, privilege for the same is uncommon for $\mathrm{BPH}$ in the current scenario.

Despite the high level of literacy among our study population, the major demerit observed was that, the health literacy of BPH was found to be extremely poor. Subsequent improvement in medication adherence, QoL, knowledge, attitude and practice were observed following an effective counselling session provided during the course of the current research. Hence, the provision of effective counselling was found to have a profound impact on promoting better and positive therapeutic outcomes.

\section{ACKNOWLEDGEMENTS}

Authors would like to thank Dr. JS Joy Jyothis, Hon. Senior consultant, Department of Urology, who helped us in collecting the data for this study.

Funding: No funding sources Conflict of interest: None declared

Ethical approval: The study was approved by the Institutional Ethics Committee

\section{REFERENCES}

1. American Urological Association Guideline: management of benign prostatic hyperplasia (BPH). 2010. Available at: http://www.auanet.org/guidelines/benign-prostatichyperplasia-(2010-reviewed-and-validity-confirmed2014). Accessed on 10 May 2020.

2. EAU Guidelines. Edn. presented at the EAU Annual Congress Amsterdam 2020. Available at: https://uroweb.org/guideline/urologicalinfections/?type=archive. Accessed on 10 May 2020.

3. Martinez M, Satheesh M. Prostate disease. Roger Walker, Cate Whittlesea. Clinical pharmacy and therapeutics. London, Churchill Livingston. Elsevier. 2012:3:753-9

4. Mary L, Roohollah S. Benign Prostatic Hyperplasia. Joseph $\mathrm{T}$ Dipiro et al. Pharmacotherapy: A Pathophysiological Approach. 10th ed. New York, Mc GrawHill. 2017:792-833.

5. Roehrborn C, McConnell J. Etiology, pathophysiology, epidemiology and natural history of benign prostatic hyperplasia. In: Walsh $\mathrm{P}$, Retik A, Vaughan E, Wein A (eds). Campbell's Urology, 8th edn. Saunders: Philadelphia, 2002;8:1297-336.

6. Kirby R, McConnell JD, Fitzpatrick JM. Griffiths K: Molecular control of prostate growth (Eds), Textbook of Benign Prostatic Hyperplasia. 1996:23-56

7. Mc Vary KT. AUA practice guideline committee. AUA guidelines on management of BPH, Chapter 1. Available at: www.auanet.org. Accessed on 10 May 2020.

8. Homma Y, Gotoh M, Yokoyama O, Masumori N, Kawauchi A, Yamanishi T, et al. Outline of JUA clinical guidelines for benign prostatic hyperplasia. Int J Urol. 2011;18(11):741-56.

9. Claus G. Current Medical Therapies for Men with Lower Urinary Tract Symptoms and Benign Prostatic Hyperplasia: Achievements and Limitations. Current Medical Therapies for Men with LUTS and BPH. Rev Urol J. 2008;10(1):17-23.

10. Barry MJ, Fowler FJ, O'Leary MP, Bruskewitz RC, Holtgrewe HL, Mebust WK, et al. The Measurement Committee of the American Urological Association: The American Urological Association Symptom Index for Benign Prostatic Hyperplasia. J Urol. 1992;148:1549-57.

11. Barry MJ, Fowler FJ, O'Leary MP, Bruskewitz RC, Holtgrewe HL, Mebust WK. Measuring diseasespecific health status in men with benign prostatic hyperplasia. Med Care. 1995;33:AS145-55.

12. Jack B, Claus GR, Paul S, Olivier H, Betsy M, Libby $B$ et al. Effect of Dutasteride, Tamsulosin and the combination on patient-reported quality of life and treatment satisfaction in men with moderate-to-severe benign prostatic hyperplasia: Two-year data from the combat trial. Brit J Urol Int. 2009;103:919-26.

13. Deep A, Ingle GK, Kishore J. Benign Prostatic Hyperplasia: Health Seeking Behaviour of patients at a tertiary care hospital. Aus Medic J. 2010;22(3):213. 
14. Ramesh A. Patient Counselling. G. Parthasarathi, Karin Nyfot-Hansen, Milap CN. A Textbook of Clinical Pharmacy Practice: Essential Concepts and Skills. 1st ed. Hyderabad, Orient Blackswan. 2004;1:60-71.

15. Mahajan P. Tadalafil therapy in symptomatic improvement of LUTS due to BPH and associated Erectile Dysfunction. J Medic Sci Clinic Resear. 2017;7:24577-82.

16. Nageratnam M, Latheef A. Prevalence of lower urinary tract symptoms in patients of Benign prostatic hyperplasia attending tertiary care hospital in the state of Andhra Pradesh. J NTR Univ Heal Sci. 2018;20:154-7.
17. Ramesh A, Kartheek S. Study of impact of patient education on health-related quality of life in benign prostatic hyperplasia (BPH) patients in a South Indian teaching Hospital. As J Pharmaceutic Clinic Resear. 2009;2:97-103.

Cite this article as: Johnson AT, Jayakumar V, Varghese PA, Vijayan K, Martin B. Impact of educational intervention on medication adherence, quality of life, knowledge attitude and practice among benign prostate hyperplasia patients- a prospective study. Int J Basic Clin Pharmacol 2020;9:1807-15. 


\section{ANNEXURE-I}

\section{Knowledge, attitude and practice (KAP) questionnaire for benign prostatic hypeplasia}

\section{Knowledge oriented questions}

1. What is benign prostatic hyperplasia (BPH)?
a) Enlargement of prostate
b) Infection of prostate
c) Cancer of prostate
d) None of the above.

2. In which age group BPH is most commonly seen?
a) $41-50$
b) $51-60$
c) $61-70$
d) $71-80$

3. BPH most commonly causes?
a) Kidney stones.
b) Urinary tract infections.
c) Urinary retention
d) All of the above.

4. Which of the following are the most common urinary symptom of BPH?
a) Incomplete emptying
b) Nocturia
c) Increased urgency and frequency.
d) All of the above.

5. Which of the following treatment intially used to treat $\mathrm{BPH}$ ?
a) Lifestyle modification
b) Medication
c) Surgery
d) None of the above

Attitude oriented questions

6. Do you think there is a need to consult a doctor for your problems?
a) Yes
b) $\mathrm{No}$
c) I don't know

7. Are you embarrased to talk to your doctor about your disease?
a) Yes
b) No

8. Which of the following therapist may reduce yours bothers?
a) Lifestyle modification
b) Medication
c) Surgery
d) None of the above

9. Do you take prescribed medicines regularly?
a) Yes
b) No

10. Do you know how does your drugs act?
a) Yes
b) No 


\section{Practice oriented questions}

11. Do you think that your drugs have some side-effects?
a) Yes
b) No

12. Do you alter doses or frequency of medications based on your symptoms?
a) Yes
b) No

13. Do you think there is a need to maintain diet control for your disease?
a) Yes
b) No
c) I don't know

14. Do you think that there is a need to avoid taking fluids during bedtime?
a) Yes
b) $\mathrm{No}$
c) I don't know

15. Do you think that alcohol consumption can aggravate your symptoms?
a) Yes
b) No
c) I don't know 